\title{
Meeting report \\ VIII International Congress of the Metastasis Research Society
}

Gill Taylor* and Philip Rudland ${ }^{+}$

*Breast Cancer Research, London, UK

†School of Biological Sciences, University of Liverpool, Liverpool, UK

Correspondence: Gill Taylor, In-house Editor, Breast Cancer Research, 34-42 Cleveland Street, London, W1T 4LB, UK. Tel: +44 (0)20 7323 0323; fax: +44 (0)20 7580 1938; e-mail; gill.taylor@breast-cancer-research.com

Received: 29 September 2000

Revisions requested: 5 October 2000

Revisions received: 9 October 2000

Accepted: 9 October 2000

Published: 2 November 2000
Breast Cancer Res 2001, 3:49-54

(C) BioMed Central Ltd on behalf of the copyright holder (Print ISSN 1465-5411; Online ISSN 1465-542X)

\section{Introduction}

This millennium meeting was held at Imperial College, London, UK, 24 ${ }^{\text {th }}-27^{\text {th }}$ September 2000. Celebrating the return of this biannual meeting to the city of its very first meeting, 300 participants from around the world attended plenary lectures, a workshop session and presented 150 posters over two sessions.

Two award lectures were given: the Paget-Ewing award lecture was given by Isaiah Fidler (University of Texas MD Anderson Cancer Center, Houston, USA) who gave an overview of the recent developments in the "seed and soil" hypothesis; Stephen Carter (Sugen, USA) gave the Kurt Hellmann award lecture, a new award in honour of those who have made an outstanding contribution in translating the problems of research in metastatic disease from the laboratory to the clinic.

The opening session consisted of a workshop entitled "Novel technologies and bioinformatics". Lance Liotta (NIH, Bethesda, USA) and Emmanual Petricoin (Center for Biological Evaluation and Research, FDA, Bethesda, USA) gave an overview of the advances that can be applied to molecular analysis of tissues, enabling thousands of molecular events to be studied simultaneously. The problem of the multistep polygenetic phenotype with more than one metastasis gene or predictive marker requires the development of technology that is capable of studying simultaneous multiple events. Techniques such as laser capture microdissection (LCM) to isolate the neoplastic cells followed by analysis using a combination of 2D gel electrophoresis and surface-enhanced laser desorption and ionisation (SELDI) can be used to investigate the complex protein patterns involved in metastasis as well as the traditional cDNA microarrays. Not unexpectedly, the changes identified cluster into four groups concerned with unrestrained growth, motility, invasion, evasion of host defenses and angiogenesis.

\section{Genes and environment}

Donald Ingber (Harvard Medical School, Boston, USA) introduced the session by reviewing work on how biochemical pathways may be modified by the structure of a cell both by its internal structure (principally the cytoskeleton) and by external structures in the microenvironment surrounding a cell. The extracellular-intracellular tensions are largely maintained by integrins acting as a bridge between these structures. Reactions inside cells take place in the solid phase with interacting molecules bound either directly or indirectly to the cytoskeletal scaffold. For example, in protein synthesis mRNAs are localised to vertices of the cytoskeleton, if tension imposed by the integrins is prevented, protein synthesis is inhibited. Likewise transcription of early genes are activated when integrin ligands bind to receptors. For many growth and differentiation signals, the growth factor/receptor, integrins and shape of the cell all have to work together and it is this architectural balance which controls developmental processes. Disturbance of this architectural balance can lead to diseases like cancer.

Jean-Paul Thiery (Institut Curie, Paris, France) continued this theme of cell shape discussing how the shape-change or plasticity of epithelial cells converting to motile fibroblastic-like cells in a malignant bladder cell model could be important in the development of cancer. This conversion could be reversibly triggered by hepatocyte growth factor/scatter factor (HGF/SF) acting through c-Src and 
the ras-MAPKinase pathway. One of the end products of these pathways is a transcription factor called slug, related to snail in Drosophila, which is associated with loss of desmosomes from the epithelial cells during this conversion process. The epithelial to fibroblastic-like reversible conversion can also be triggered by structural components outside the cell such as native collagens and laminin 5 . Thus type I collagen induces specific tyrosine phosphorylation of focal adhesion kinase (FAK) and paxillin, two proteins present in focal adhesions. Therefore a common focal point of SF and extracellular architectural control may have been identified. Overexpression of fibroblast growth factor-1 (FGF-1) or FGF-2 in these malignant bladder epithelial cells converts them permanently into the fibroblast-like cell type endowed permanently with motile, invasive and metastatic properties. When the released FGF-1 and FGF-2 are inhibited, the angiogenic response is blocked, but not the fibroblast-like cell shape conversion. It was originally proposed that the $18 \mathrm{KDa}$ form of FGF-2 was responsible for its autocrine and paracrine signalling effects, but subsequently a nuclear $24 \mathrm{KDa}$ moiety was shown to be responsible for the epithelial to fibroblast conversion and for the spontaneous metastasis to lungs, but not for the angiogenic response.

The next two speakers turned to the identification of metastasis-suppressor genes. Carrie Rinker-Schaeffer (University of Chicago, USA) used the Dunning metastatic prostatic cancer cell line in rats and microcell transfer to identify regions of human chromosomes that could suppress its metastatic activity in rats. Suppressor activity could be identified on human chromosomes 7, 8, 10, 12, 16 and 17 but not on chromosome 6 . The suppressor activity on chromosome 17 was shown to localise to the MAPKinase kinase 4/stress activated erk kinase (MKK4/SEK1). In contrast to the chromosome 12 suppressor, where no prostatic carcinoma cells were found in the lungs in the metastatic assay, MKK4 (chromosome 17) suppressed cells were found in small numbers in the lung. It was suggested that MKK4 blocked a growth or cell survival signal that was specifically required in the lungs, since none of the standard in vitro tests for growth, transformation, cell motility or angiogenesis showed differences in the MKK4 transfected cells. Loss of heterozygocity analyses in human carcinoma suggested that suppressors existed in similar regions on chromosome 12 and 17.

Andrea McClatchey (MGH Cancer Center, Charlestown, USA) then discussed the function of the neurofibromatosis type 2 (NF2) suppressor, merlin, in tumorigenesis and metastasis. Merlin is a member of the ezrin, radixin, moesin (ERM) family of cytoskeleton-membrane linkers that are thought to maintain and reorganise the cytoskeleton. NF2 ${ }^{-}$ mutant mice were generated and mice heterozygous for this mutation developed osteosarcomas, fibrosarcomas and liver carcinomas that exhibited loss of the remaining NF2 gene. Those cancers were all highly metastatic to the lung and liver. Analysis of the NF2- cells revealed changes in cell migration, invasion and survival. In normal cells phosphorylation of merlin, which can be induced by Rac, is associated with growth arrest. Moreover, overexpression of merlin inhibits aspects of Rac signalling and in the NF2- cells aspects of Rac-mediated signalling are increased. It would appear that merlin sits at the cytoskeleton-membrane interface, and its phosphorylation status causes it to shuttle to and from this point, thus allowing regulation of the cytoskeleton, perhaps to produce the plasticity required for metastatic cells, as discussed by earlier speakers.

\section{Gene expression, host modulation}

The search for genes and proteins associated with the metastatic processes, either in a positive or a negative way, continued in oral presentations by members in the Gene Expression-Host Modulation Section. Danny Welch (Penn State University, Pennsylvania, USA) identified genes which changed in expression after transfer of human chromosome 11 which altered a low/moderately metastatic breast cancer cell line into a highly metastatic one in nude mice. The highest increase in expression was observed in osteopontin and then more modest increases in a ras homologue, osteonectin, calcyclin, casein kinase and tenascin. He also identified 4 potential suppressors, $\alpha 1$-antichymotrypsin, PEDF, Kai 1 and a novel BRMS-1 (breast metastasis suppressor-1). BRMS-1 failed to affect tumour growth, was present in the region close to the gene for cyclin D1 on chromosome 11 which was modified in $75 \%$ of human breast carcinomas, has casein kinase and protein kinase $\mathrm{C}$ phosphorylation sites, and a sequence consistent with a leucine zipper transcriptional complex repressor. In biological assays BRMS-1 restored gap junction communication in suitably transfected cells.

Erica Sloan (Peter MacCallum Cancer Institute, Melbourne, Australia) using cDNA microarray and candidate gene approaches in genetically matched mouse mammary tumour sublines that do or do not metastasize to bone, found reproducible changes in the levels of caveolin-1 (involved in cholesterol metabolism), protease nexin I, beta 3 integrin and parathyroid hormone-related protein (PTHrP). Of the 15 genes initially identified from 5000 genes only 4 scanned positively. Some like PTHrP are not a primary determinant of metastasis to bone but can modulate the extent of tumour burden. Mohamed El-Tanani (University of Liverpool, UK) reported on a system whereby small fragments of DNA from breast carcinoma cell lines can upregulate the production of osteopontin and thereby cause a benign rat mammary cell line to metastasize when introduced into the mammary fat pad of syngeneic rats. This effect is accomplished, in part, by sequestering an inhibitory transcription factor Tcf-4. These results demonstrate that the transcription factor at the end of the Wnt signalling pathway can modify the production 
of a protein, osteopontin, important in the metastatic process, at least in animal models, and suggests that the Wnt signalling pathway could be important in breast, as it is in the development of colon cancer.

In addition to the suppressors of metastasis which seemed to dominate the non mammary gland areas, the metastasispromoting molecules were to the fore in the mammary areas. Thus inhibition of the tumour metastasis-associated human MTA1 gene (Garth Nicolson, Institute of Molecular Medicine, Huntington Beach, USA) and the metastasisassociated S100A4 gene (Berit Mathisen, University of Tromso, Norway) by targeted antisense and ribozyme technologies, respectively, showed that they could block metastatic potential. MTA1 probably functions as a transcription factor involved in cell signalling whereas one of the effects of S100A4 may be to activate matrix metalloproteinases (MMPs). The control of the transcription of these types of molecules is actively being pursued. For example the expression of the tumour metastasis suppressor nm23 was shown to be controlled by the transcription factor CTF/NF1-X and two novel mammary-specific transcription factors (Taoufik Ouatas, National Cancer Institute, Bethesda, MD, USA) and the S100A4 could be induced by interferon- $\gamma$ possibly acting through the STAT1 transcription factor (Kristin Anderson, Norwegian Radium Hospital, Oslo, Norway). Immunocytochemical techniques also highlighted the presence of S100A4 in human breast cancer (Kjetil Pedersen, Norwegian Radium Hospital, Oslo, Norway) (Philip Rudland, University of Liverpool, Liverpool, UK).

\section{Preclinical models/clinical studies}

George Naumov (London Regional Cancer Center, London, Canada), a young investigator award winner, reported on an elegant model for dormancy in breast cancer, in which the fate of high and low metastatic mouse mammary carcinoma cell lines could be followed using endogenous-labelling with green fluorescent protein (GFP) and exogenously-labelled fluorescent nanospheres to quantitate cellular recovery. Following injections into the mesenteric vein, 50\% of the low metastatic cell line survived as single cells 77 days later, and even $20 \%$ of the high metastatic cell line remained as single cells 21 days later in the presence of large experimental metastases. The results in this mammary system and from other nonmammary systems suggested that apparently nonmetastatic cells could survive for long periods of time in secondary organs of the host. It was the ability of the cancer cells to grow into a large micrometastasis prior to vascularisation that was one of the more critical steps in the development of secondary tumours.

The importance of micrometastases was continued on the human scale in this session. Oystein Fodstad (Norwegian Radium Hospital, Oslo, Norway) made a comparison of different methods to detect micrometastases on cytospins of bone marrow and blood, from inter alia breast cancer patients using immunomagnetic selection. His cell immunobead detection method gave the best results enabling 10 times more mononuclear cells to be screened $\left(\sim 2 \times 10^{7}\right)$ compared to conventional immunocytochemical detection methods. In many cases where one immunocytochemically positive tumour cell was found, immunorosetting proved to be negative. There was moreover a good correlation with detection of micrometastases by the immunobead detection technique and clinical data. A similar presentation describing the advantages of using immunomagnetic methods for the isolation of circulating cancer cells from prostatic carcinoma patients was given by Sat Bhattacharya (Memorial Sloan-Kettering Cancer Centre, New York, USA). He also suggested that the use of multiple markers greatly enhances the molecular detection of circulating prostatic carcinoma cells.

\section{Adhesion, motility, proteases}

This session was opened with a keynote lecture by Hynda Kleinman (NIDCR, National Institutes of Health, Bethesda, USA) who discussed the importance of the basement membrane in not being just a physical barrier, but actively enhancing tumour growth, by harbouring angiogenic, metastatic and tumorigenic factors. Laminin, one of the most abundant components of the basement membrane, is especially prominent in influencing tumour growth by stimulating protease activity, regulating angiogenesis and tumour cell migration and growth. This group have performed a peptide screen of laminin-1 to identify those peptides responsible for its action. Two synthetic peptides, A13 and C16, were identified on two different chains of the laminin molecule. These peptides increased cell adhesion and migration; C16 activated MMP9 in melanoma cells and both were angiogenic in chick chorioallantoic membrane assays. They therefore possessed the ability to promote properties associated with malignant cells. The receptor for these peptides was identified as integrins $\alpha 5 \beta 1$ and $\alpha v \beta 3$. The sequences for these peptides can be found in 10/12 of the known isoforms of laminin, inferring a critical role in the biological activity of laminin.

Kenneth Van Golen (University of Michigan Comprehensive Cancer Center, Ann Arbor, USA) described the identification of two genes that are consistently altered in the aggressive disease, inflammatory breast cancer (IBC), one gene named LIBC (lost in IBC) and the gene for RhoC GTPase (a member of the Ras superfamily). Human mammary epithelial cells that stably overexpress RhoC GTPase were generated and tested in a variety of assays. The cells showed enhanced invasive and motile properties and, when examined by electron microscopy, exhibited a highly polarised cell structure thus confirming the motile potential of the cell. When the cells were injected into nude mice, tumours formed in $25 \%$ of the mice with $10 \%$ developing micrometastases, suggesting that RhoC overexpression can contribute to the metastatic phenotype. 
In a provocative lecture, Ruth Muschel (University of Pennsylvania, Philadelphia, USA) challenged the mainstream view of the mechanism by which metastases form. Instead of the hypothesis of tumour cells becoming 'stuck' in the small blood vessels with subsequent extravasation leading to colony formation in the adjacent organ, she proposed that metastatic cells attach to capillaries with intravascular proliferation and colony formation subsequently occurring in the adjacent organ. By injecting GFP-labelled tumour cells into mouse tail veins, and subsequent visualisation of the pulmonary circulation by staining the blood vessels themselves, she observed colony formation intravascularly, in which extravasation was a rare event. This model of intravascular metastasis suggests that early colonies would be especially vulnerable to intravascular drugs.

\section{Signalling, cytoskeleton, motility}

lan Hart (Rayne Institute, London, UK) described studies investigating the receptor for hyaluronic acid mediated motility (RHAMM), originally thought to be a membrane bound protein and present in human tumour cells. However, this protein has actually been shown to be an intracellular protein and thus a name change to intracellular hyaluronic acid binding protein (IHABP) has been suggested. RHAMM has two microtubule binding domains and is also capable of binding to actin filaments thus potentially having a role in tumour cell motility. Using a polyclonal antibody, lobular and ductal mammary carcinomas were examined for RHAMM expression. There was significantly lower expression of RHAMM in the cytoplasm of cells, but a higher expression in the trabeculae and invasive edge of the tumour islands. Thus RHAMM could prove important in contributing to the invasiveness of tumour cells.

Paulo Comoglio (University of Torino, Candiolo, Italy) reported on the mesenchyme-produced HGF/SF which, working through the MET receptor, is able to accomplish apparently independent biological responses: cell proliferation, survival, motility/invasion, and cell polarity, leading normally to invasive cell growth. This induces branching morphogenesis (tubulogenesis) on mammary epithelial cells. MET and its two closely related family members, RON and SEA, feature unique signal transduction properties, as their cytoplasmic tails contain a two-tyrosine multifunctional docking site that binds multiple $\mathrm{SH} 2$-containing intracellular signal transducers. Dissection of the signalling pathways has shown that ras, PI3-K and STAT were responsible for growth, scattering/survival and polarity/ morphogenesis, respectively. PI3-K operated through Rac for scattering leading to cell motility/invasion and through Akt for apoptosis protection (anoikis). Dr Comoglio also reported on the discovery of the Sex/plexins, sharing homology with the extracellular domain of MET and their ligands, the semaphorins, which in mammalian cells control cell-cell repulsion, a process reminiscent of "scattering". Thus the name of the original receptor MET may not be inappropriate for a class of large receptors producing invasive growth in diverse situations.

\section{Multiple functions of proteases}

This session emphasised that tumour initiation is not just caused by a breakdown of connective tissue but involves many levels and interactions between multiple cell types.

Lynn Matrisian (Vanderbilt University, Nashville, USA) began by contesting the hypothesis that MMPs are only involved in degradation of the basement membrane and extracellular matrix (ECM). She showed that Matrilysin (MMP 7), unlike other MMPs, is virtually always expressed in epithelial cells and has been shown to cleave membrane bound Fas Ligand (Fas L), releasing soluble apoptosis-inducing Fas L. However, when normal murine mammary gland cells were stably transfected with matrilysin, a reduced sensitivity to Fas-mediated apoptosis in response to matrilysin treatment was demonstrated. This reduced sensitivity to apoptosis-inducing agents could contribute to tumour progression by the development of cells that are less likely to undergo apoptosis for example in response to DNA damage.

Bill Stetler-Stevenson (NCl, Bethesda, USA) followed with the conflicting roles of tissue inhibitors of matrix metalloproteinases (TIMPs). TIMPs appear to act as enhancers of cell growth in tumour cells and fibroblasts but inhibit the growth of cultured endothelial cells. TIMPs can directly inhibit FGF and VEGF stimulated growth of endothelial cells thus acting as an angiogenesis inhibitor. In trying to elucidate the conflicting roles of TIMPs, this group have studied the effects of TIMP on a human B cell lymphoma model. When grown subcutaneously in nude mice, TIMP-1 transfected cells initially have an accelerated growth, however at later times the tumours necrose and regress due to the anti-angiogenic effects of TIMP-1 on endothelial cells. In a second model system using a TIMP-2 mutant devoid of MMP inhibitor activity they demonstrated that TIMP-2 inhibition of angiogenesis was independent of MMP inhibition. These results suggest that TIMPs can modify cell growth independently of MMP activity.

The role of heparanase was the subject of the lecture by Israel Vlodaysky (Hadassah University Hospital, Jerusalem, Israel). The heparanase gene is found in highly metastatic human breast cancer cells, but not in normal epithelial cells. In biopsy specimens, immunocytochemical staining for heparanase was especially pronounced in invasive ductal mammary carcinomas. Overexpression of heparanase leads to increased metastasis and mortality in mice. It has been postulated that heparanase may release growth factors such as FGF from the surrounding tissue (eg basement membrane) thus initiating angiogenesis and contributing to tumourigenesis. In transgenic mice that overexpress the heparanase gene, staining is seen in the 
mammary gland and could possibly represent a premalignant state. Knock out animal models of this gene are being developed and will provide an indication of the importance of heparanase.

\section{Molecular crosstalk and angiogenesis}

Following the plenary lecture by Mary Hendrix (University of lowa, lowa City, USA), in which her work on vasculogenic mimicry was elegantly explained [1], the main theme of this session was the subject of inhibitors of angiogenesis and therapies.

The antiangiogenic property of thrombospondin-1 (TSP-1) was described by Giulia Taraboletti (Mario Negri Institute, Bergamo, Italy). In a series of experiments, it was shown that TSP-1 inhibits the binding of FGF-2 to its low affinity receptors present on endothelial cells. TSP-1 also released ECM bound FGF-2 as a TSP-1/FGF-2 complex, which has subsequently been shown to stimulate endothelial cell proliferation. Thus, by reducing the actions of TSP-1 on the ECM, this could represent another potential anti-angiogenic mechanism for TSP-1.

The role of oxygen radicals in angiogenesis was addressed by Moshe Marikovsky (Hebrew University, Jerusalem, Israel). Oxygen radicals are a by-product of normal cell metabolism. Mice overexpressing the enzyme superoxide dismutase (SOD-1) showed increased angiogenesis following FGF administration. This effect was attenuated by disulfiram, a specific inhibitor of SOD-1. Disulfiram also induced apoptosis in cultured endothelial cells, indicating its possible mechanism of action. It has been shown that proliferating endothelial cells are a target for oxygen radicals and thus have increased levels of SOD-1. These results imply that targeting the SOD-1 pathway may have potential for inhibition of metastasis.

GFP labelled tumour cells were utilized in the in vivo studies by the group of Robert Hoffman (Anticancer Inc, San Diego, USA). When these labelled cells were injected into the tail vein of mice, the growth of the tumour cells and metastases were clearly visable by whole-body imaging. Vascularisation could be detected by the contrast of the dark vessels against the highlighted fluorescent tumours. This system was used to study transplanted Lewis lung carcinoma cells, and tracked the tumour cells crossing the diaphragm and using the lymphatic system to travel to the contralateral lung. This system could potentially be useful in the study of angiogenesis inhibitors.

Sofia Mejaver (University of Michigan Medical Center, Ann Arbor, USA) described investigations into the anti-angiogenic properties of copper deficiency. Tetrathiomolybdate (TM), a copper chelator approved for human use, was administered to female MMTV-HER2/neu transgenic mice that had developed large mammary tumours. Administra- tion of TM arrested the growth of these tumours, therefore implying that copper deficiency inhibits de novo clinically significant mammary tumours. Clinical trials of TM have begun, showing some initial promising results, albeit in a very small number of patients.

\section{Molecular therapeutics}

William Fogler (Entremed Inc, Rockville, USA) described the latest developments on the role of endostatin and its potential as an anti-angiogenesis therapy. Phage display techniques were utilized to elucidate the molecular target of endostatin. A binding protein sharing epitopes with tropomyosin type 3 was identified. It was also shown that endostatin and tropomyosin colocalise to the myofilaments and there is a physical interaction between these two in cultured human umbilical vein endothelial cells, although it remains to be determined whether this truly represents the site of action of endostatin.

Dennis Slamon (UCLA, Los Angeles, USA) gave the final talk and highlight of this session which encompassed both the discovery of the overexpression of the growth factor receptor c-erbB-2 in 25-30\% of patients with invasive breast cancer and the use of the anti-HER-2/neu humanised antibody Herceptin in mouse and in man. The major Phase III trial in 469 c-erbB-2-positive patients comparing the best available standard therapy versus best plus Herceptin showed that the latter improved objective response rates by $54 \%$, response duration by $58 \%$ and time to progression by $65 \%$. Preliminary analysis showed an improved 1 year survival of $11 \%$ and at 30 months, $25 \%$. There was little change with Herceptin alone. These figures may be improved if the selection criterion per patient is based on gene amplification rather than overexpression of c-erbB-2. The major problem in its use with anthracyclines was cardiac toxicity in almost $40 \%$ of women, but this may be reduced by combining Herceptin with other agents. The actual mechanism of Herceptin does not appear to depend on the immune system since it is active in immunodeficient mice and removal of its $\mathrm{Fc}$ portion fails to inhibit its effects. It is thought that Herceptin blocks dimerisation/oligermerisation of receptors causing aberrant intracellular signalling, but the precise mechanism has yet to be elucidated. Herceptin is therefore one of the first biological response modifiers to have shown some clinical success and as such has led to its approval in the treatment of patients with c-erbB-2 overexpressing breast cancers.

Angiogenesis: conundrums and controversies Roy Bicknell (ICRF, Molecular Oncology Laboratories, Oxford, UK) continued on the theme of oxygen free radical stress by reporting on the actions of thymidine phosphorylase (TP). TP is an angiogenic factor, first isolated from platelets, and has been shown to be elevated in the serum of cancer patients. It is strongly overexpressed 
in carcinomas, including breast. To elucidate the mechanism of action of TP, expression of haem-oxygenase, a marker of oxidative stress, was examined in carcinoma cells. TP increased haem-oxygenase levels, thus inducing oxidative stress by the generation of free radicals. Subsequently, secretion of angiogenic factors such as vascular endothelial growth factor and interleukin-8 are increased and MMPs are induced, ultimately resulting in the formation of blood vessels.

\section{Conclusion}

The VIII International Congress of the Metastasis Research Society terminated where it began in London 20 years earlier. Kurt Hellmann (St John's Institute of Dermatology, London, UK), one of the founding members of the Society, commented that in the beginning there was little interest in the study of metastatic processes per se. In those days the major research efforts were concentrated on unravelling the uncontrolled growth of tumours. This conference, however, demonstrated that metastasis research is now actively pursued throughout the world, in various guises, as the key to producing potentially new therapies directed at eradicating the major forms of cancer. Research on the uncontrolled growth of tumours has spread, it would appear, to almost all aspects of developmental biology not only to the cancer cells themselves, but also to the host organs in which the metastases develop. Thus the "seed and soil" hypothesis first expounded by Paget more than a century ago for the process of metastasis, is now coming to fruition, as outlined by Dr Fidler in his Award Lecture. The generation of genomic instability followed by selection of cells capable of undergoing the steps of the metastatic cascade means that parallel pathways with perhaps different balances of regulatory molecules may underlie the overall process, even for a cancer like that of invasive ductal carcinoma of the breast. The use of modern genomic and proteomic techniques to unravel the potential multitude of molecules involved in the metastatic cascade is now proving invaluable. The new systemic drugs that are being developed against metastatic disease are targeting and will continue to target the new regulatory molecules as they are discovered. However, many of these new drugs are not cytotoxic but rather cytostatic in nature. Thus the question raised by Dr Carter in his Award Lecture as to whether the cytotoxic endpoint of objective response can be used for decision making in the development of such therapies is pertinent to this area of research. One felt on leaving this Conference that, unlike many cancer conferences, a rational view on how cancer could spread had been presented. Whether the plethora of results presented at this conference is relevant to specific human cancers and can be utilised to produce successful treatments of metastatic disease only time will tell.

\section{Reference}

1. Hendrix MJC, Seftor EA, Kirschmann DA, Seftor REB: Molecular expression of vascular markers by aggressive breast cancer cells. Breast Cancer Res 2000, 2:417-422 